\title{
THE DEQUANTIZATION TRANSFORM AND GENERALIZED NEWTON POLYTOPES
}

\author{
G. L. LITVINOV AND G. B. SHPIZ
}

\begin{abstract}
For functions defined on $\mathbf{C}^{n}$ or $\mathbf{R}_{+}^{n}$ we construct a dequantization transform $f \mapsto \hat{f}$; this transform is closely related to the Maslov dequantization. If $f$ is a polynomial, then the subdifferential $\partial \hat{f}$ of $\hat{f}$ at the origin coincides with the Newton polytope of $f$. For the semiring of polynomials with nonnegative coefficients, the dequantization transform is a homomorphism of this semiring to the idempotent semiring of convex polytopes with respect to the well-known Minkowski operations. Using the dequantization transform we generalize these results to a wide class of functions and convex sets.
\end{abstract}

\section{Introduction. The Maslov Dequantization and the Dequantization TRANSFORM}

Let $\mathbf{R}$ and $\mathbf{C}$ be the fields of real and complex numbers. The well-known max-plus algebra $\mathbf{R}_{\max }=\mathbf{R} \cup\{-\infty\}$ is defined by the operations $x \oplus y=\max \{x, y\}$ and $x \odot y=x+y$.

The max-plus algebra can be treated as a result of the Maslov dequantization of the semifield $\mathbf{R}_{+}$of all nonnegative numbers, see, e.g., 1, 2]. The change of variables

$$
x \mapsto u=h \log x,
$$

where $h>0$, defines a map $\Phi_{h}: \mathbf{R}_{+} \rightarrow \mathbf{R} \cup\{-\infty\}$. Let the addition and multiplication operations be mapped from $\mathbf{R}_{+}$to $\mathbf{R} \cup\{-\infty\}$ by $\Phi_{h}$, i.e. let

$$
\begin{gathered}
u \oplus_{h} v=h \log (\exp (u / h)+\exp (v / h)), \quad u \odot v=u+v, \\
\mathbf{0}=-\infty=\Phi_{h}(0), \quad \mathbf{1}=0=\Phi_{h}(1) .
\end{gathered}
$$

It can easily be checked that $u \oplus_{h} v \rightarrow \max \{u, v\}$ as $h \rightarrow 0$. Thus we get the semifield $\mathbf{R}_{\max }$ (i.e. the max-plus algebra) with zero $\mathbf{0}=-\infty$ and unit $\mathbf{1}=0$ as a result of this deformation of the algebraic structure in $\mathbf{R}_{+}$.

The semifield $\mathbf{R}_{\max }$ is a typical example of an idempotent semiring; this is a semiring with idempotent addition, i.e., $x \oplus x=x$ for arbitrary element $x$ of this semiring, see, e.g., [3].

2000 Mathematics Subject Classification. Primary 81Q20, 14M25; Secondary 51P05, 52A20, 52B20.

Key words and phrases. The Maslov dequantization, the dequantization transform, Newton polytopes, subdifferential, convex sets, sublinear functions.

This work has been supported by the RFBR grant 02-01-01062 and the Erwin Schrödinger International Institute for Mathematical Physics (ESI). 
The analogy with quantization is obvious; the parameter $h$ plays the role of the Planck constant [2]. In fact the Maslov dequantization is the usual Schrödinger dequantization but for pure imaginary values of the Planck constant (see, e.g., 4]). The map $x \mapsto|x|$ and the Maslov dequantization for $\mathbf{R}_{+}$give us a natural passage from the field $\mathbf{C}$ (or $\mathbf{R}$ ) to the max-plus algebra $\mathbf{R}_{\max }$.

Let $X$ be a topological space. For functions $f(x)$ defined on $X$ we shall say that a certain property is valid almost everywhere (a.e.) if it is valid for all elements $x$ of an open dense subset of $X$.

Suppose $X$ is $\mathbf{C}^{n}$ or $\mathbf{R}^{n}$; denote by $\mathbf{R}_{+}^{n}$ the set $x=\left\{\left(x_{1}, \ldots, x_{n}\right) \in X \mid x_{i} \geq\right.$ 0 for $i=1,2, \ldots, n\}$. For $x=\left(x_{1}, \ldots, x_{n}\right) \in X$ we set $\exp (x)=\left(\exp \left(x_{1}\right), \ldots\right.$, $\left.\exp \left(x_{n}\right)\right)$; so if $x \in \mathbf{R}^{n}$, then $\exp (x) \in \mathbf{R}_{+}^{n}$.

Denote by $\mathcal{F}\left(\mathbf{C}^{n}\right)$ the set of all functions defined and continuous on an open dense subset $U \subset \mathbf{C}^{n}$ such that $U \supset \mathbf{R}_{+}^{n}$. In all the examples below we consider even more regular functions, which are holomorphic in $U$. It is clear that $\mathcal{F}\left(\mathbf{C}^{n}\right)$ is a ring (and an algebra over $\mathbf{C}$ ) with respect to the usual addition and multiplications of functions.

For $f \in \mathcal{F}\left(\mathbf{C}^{n}\right)$ let us define the function $\hat{f}_{h}$ by the following formula:

$$
\hat{f}_{h}(x)=h \log |f(\exp (x / h))|,
$$

where $h$ is a (small) real parameter and $x \in \mathbf{R}^{n}$. Set

$$
\hat{f}(x)=\lim _{h \rightarrow 0} \hat{f}_{h}(x),
$$

if the right-hand part of (3) exists almost everywhere. We shall say that the function $\hat{f}(x)$ is a dequantization of the function $f(x)$ and the map $f(x) \mapsto \hat{f}(x)$ is a dequantization transform. By construction, $\hat{f}_{h}(x)$ and $\hat{f}(x)$ can be treated as functions taking their values in $\mathbf{R}_{\text {max }}$. Note that in fact $\hat{f}_{h}(x)$ and $\hat{f}(x)$ depend on the restriction of $f$ to $\mathbf{R}_{+}^{n}$ only; so in fact the dequantization transform is constructed for functions defined on $\mathbf{R}_{+}^{n}$ only. It is clear that the dequantization transform is generated by the Maslov dequantization and the map $x \mapsto|x|$. Of course, similar definitions can be given for functions defined on $\mathbf{R}^{n}$ and $\mathbf{R}_{+}^{n}$.

We shall see that if $f(x)$ is a polynomial, then there exists the dequantization $\hat{f}$ of this polynomial and the subdifferential $\partial \hat{f}$ of the function $\hat{f}$ coincides with the Newton polytope of the polynomial $f$.

It is well known that all the convex compact subsets in $\mathbf{R}^{n}$ form an idempotent semiring $\mathcal{S}$ with respect to the Minkowski operations: for $A, B \in \mathcal{S}$ the sum $A \oplus B$ is the convex hull of the union $A \cup B$; the product $A \odot B$ is defined in the following way: $A \odot B=\{x \mid$ $x=a+b$, where $a \in A, b \in B\}$. In fact $\mathcal{S}$ is an idempotent linear space over $\mathbf{R}_{\text {max }}$ (see, e.g., [4]). Of course, the Newton polytopes in $V$ form a subsemiring $\mathcal{N}$ in $\mathcal{S}$. If $f, g$ are polynomials, then $\partial(\widehat{f g})=\partial \hat{f} \odot \partial \widehat{g}$; moreover, if $f$ and $g$ are "in general position", then $\partial(\widehat{f+g})=\partial \hat{f} \oplus \partial \widehat{g}$. For the semiring of all polynomials with nonnegative coefficients the dequantization transform is a homomorphism of this "traditional" semiring to the idempotent semiring $\mathcal{N}$. 
Our aim is to prove these results and to generalize them to a more extensive class of functions and convex sets.

The authors are grateful to A. N. Sobolevskil for his help and useful discussions.

\section{The DEQUANTIZATION TRANSFORM: ALGEBRAIC PROPERTIES}

Denote by $V$ the set $\mathbf{R}^{n}$ treated as a linear Euclidean space (with the scalar product $\left.(x, y)=x_{1} y_{1}+x_{2} y_{2}+\cdots+x_{n} y_{n}\right)$ and set $V_{+}=\mathbf{R}_{+}^{n}$. We shall say that a function $f \in \mathcal{F}\left(\mathbf{C}^{n}\right)$ is dequantizable whenever its dequantization $\hat{f}(x)$ exists (and is defined on an open dense subset of $V)$. By $\mathcal{D}\left(\mathbf{C}^{n}\right)$ denote the set of all dequantizable functions and by $\widehat{\mathcal{D}}(V)$ denote the set $\left\{\hat{f} \mid f \in \mathcal{D}\left(\mathbf{C}^{n}\right)\right\}$. Recall that functions from $\mathcal{D}\left(\mathbf{C}^{n}\right)$ (and $\widehat{\mathcal{D}}(V)$ ) are defined almost everywhere and $f=g$ means that $f(x)=g(x)$ a.e., i.e., for $x$ ranging over an open dense subset of $\mathbf{C}^{n}$ (resp., of $V$ ). Denote by $\mathcal{D}_{+}\left(\mathbf{C}^{n}\right)$ the set of all functions $f \in \mathcal{D}\left(\mathbf{C}^{n}\right)$ such that $f\left(x_{1}, \ldots, x_{n}\right) \geq 0$ if $x_{i} \geq 0$ for $i=1, \ldots, n$; so $f \in \mathcal{D}_{+}\left(\mathbf{C}^{n}\right)$ if the restriction of $f$ to $V_{+}=\mathbf{R}_{+}^{n}$ is a nonnegative function. By $\widehat{\mathcal{D}}_{+}(V)$ denote the image of $\mathcal{D}_{+}\left(\mathbf{C}^{n}\right)$ under the dequantization transform. We shall say that functions $f, g \in \mathcal{D}\left(\mathbf{C}^{n}\right)$ are in general position whenever $\hat{f}(x) \neq \widehat{g}(x)$ for $x$ running an open dense subset of $V$.

Theorem 1. For functions $f, g \in \mathcal{D}\left(\mathbf{C}^{n}\right)$ and any nonzero constant $c$, the following equations are valid:

1) $\widehat{f g}=\hat{f}+\widehat{g}$;

2) $|\hat{f}|=\hat{f} ; \widehat{c f}=f ; \widehat{c}=0$;

3) $(\widehat{f+g})(x)=\max \{\hat{f}(x), \widehat{g}(x)\}$ a.e. if $f$ and $g$ are nonnegative on $V_{+}$(i.e., $f, g \in$ $\mathcal{D}_{+}\left(\mathbf{C}^{n}\right)$ ) or $f$ and $g$ are in general position.

Left-hand sides of these equations are well-defined automatically.

Proof. Statements 1) and 2) can be easily deduced from our basic definitions and formulas (2) and (3).

Let us prove statement 3). Set $\tilde{x}_{h}=\exp (x / h) \in V_{+}$. Suppose $f, g \in \mathcal{D}_{+}\left(\mathbf{C}^{n}\right)$; then $|f|=f,|g|=g,|f+g|=f+g$ on $V_{+}$and we have the following inequalities:

$$
\max \left\{f\left(\tilde{x}_{h}\right), g\left(\tilde{x}_{h}\right)\right\} \leq(f+g)\left(\tilde{x}_{h}\right) \leq 2 \max \left\{f\left(\tilde{x}_{h}\right), g\left(\tilde{x}_{h}\right)\right\} .
$$

Hence

$$
h \log \left(\max \left\{f\left(\tilde{x}_{h}\right), g\left(\tilde{x}_{h}\right)\right\}\right) \leq h \log \left((f+g)\left(\tilde{x}_{h}\right)\right) \leq h \log 2+h \log \left(\max \left\{f\left(\tilde{x}_{h}\right), g\left(\tilde{x}_{h}\right)\right\}\right) .
$$

But $h \log 2 \rightarrow 0$ as $h \rightarrow 0$, and the logarithmic function is monotonic. Thus we have

$$
\max \{\hat{f}(x), \widehat{g}(x)\} \leq \widehat{(f+g})(x) \leq \max \{\hat{f}(x), \widehat{g}(x)\},
$$

which completes this part of our proof.

A similar idea can be used if functions $f$ and $g$ are in general position. Without loss of generality we can suppose that $\hat{f}(x)<\widehat{g}(x)$ almost everywhere in $V$. Take an $x \in V$ where this inequality holds. In this case there exists a positive number $c$ such that $h \log \left|f\left(\tilde{x}_{h}\right)\right|+$ $c<h \log \left|g\left(\tilde{x}_{h}\right)\right|$ if the parameter $h$ is small enough. Hence $\left|f\left(\tilde{x}_{h}\right)\right| \exp (c / h)<\left|g\left(\tilde{x}_{h}\right)\right|$. 
Note that $\exp (c / h) \rightarrow \infty$ as $h \rightarrow \infty$; hence $\exp (c / h)>2$ if $h$ is small enough. Therefore we have $\left.\mid f\left(\tilde{x}_{h}\right)\right)|<(1 / 2)| g\left(\tilde{x}_{h}\right) \mid$ and $(1 / 2)\left|g\left(\tilde{x}_{h}\right)\right|<\left|f\left(\tilde{x}_{h}\right)+g\left(\tilde{x}_{h}\right)\right|$. On the other hand, we obviously have the inequality $\left|(f+g)\left(\tilde{x}_{h}\right)\right|<2\left|g\left(\tilde{x}_{h}\right)\right|$. So we get

$$
\frac{1}{2}\left|g\left(\tilde{x}_{h}\right)\right|<\left|(f+g)\left(\tilde{x}_{h}\right)\right|<2\left|g\left(\tilde{x}_{h}\right)\right|
$$

from this and from formulas (2) and (3) it follows that

$$
\widehat{g}(x) \leq \widehat{(f+g})(x) \leq \widehat{g}(x)=\max \{\hat{f}(x), \widehat{g}(x)\} .
$$

This concludes the proof.

Corollary 1. The set $\mathcal{D}_{+}\left(\mathbf{C}^{n}\right)$ has a natural structure of a semiring with respect to the usual addition and multiplication of functions taking their values in $\mathbf{C}$. The set $\widehat{\mathcal{D}}_{+}(V)$ has a natural structure of an idempotent semiring with respect to the operations $(f \oplus$ $g)(x)=\max \{f(x), g(x)\},(f \odot g)(x)=f(x)+g(x)$; elements of $\widehat{\mathcal{D}}_{+}(V)$ can be naturally treated as functions taking their values in $\mathbf{R}_{\max }$. The dequantization transform generates a homomorphism from $\mathcal{D}_{+}\left(\mathbf{C}^{n}\right)$ to $\widehat{\mathcal{D}}_{+}(V)$.

\section{Generalized polynomials And simple Functions}

For any nonzero number $a \in \mathbf{C}$ and any vector $d=\left(d_{1}, \ldots, d_{n}\right) \in V=\mathbf{R}^{n}$ we set $m_{a, d}(x)=a \prod_{i=1}^{n} x_{i}^{d_{i}}$; functions of this kind we shall call generalized monomials. Generalized monomials are defined a.e. on $\mathbf{C}^{n}$ and on $V_{+}$, but not on $V$ unless the numbers $d_{i}$ take integer or suitable rational values. We shall say that a function $f$ is a generalized polynomial whenever it is a finite sum of linearly independent generalized monomials. For instance, Laurent polynomials are examples of generalized polynomials.

As usual, for $x, y \in V$ we set $(x, y)=x_{1} y_{1}+\cdots+x_{n} y_{n}$. The following proposition is a result of a trivial calculation.

Proposition 1. For any nonzero number $a \in V=\mathbf{C}$ and any vector $d \in V=\mathbf{R}^{n}$ we have $\left(\widehat{m_{a, d}}\right)_{h}(x)=(d, x)+h \log |a|$.

Corollary 2. If $f$ is a generalized monomial, then $\hat{f}$ is a linear function.

Recall that a real function $p$ defined on $V=\mathbf{R}^{n}$ is sublinear if $p=\sup _{\alpha} p_{\alpha}$, where $\left\{p_{\alpha}\right\}$ is a collection of linear functions. Sublinear functions defined everywhere on $V=\mathbf{R}^{n}$ are convex; thus these functions are continuous, see [6], Theorem 5.5 and Corollary 10.1.1. We discuss sublinear functions of this kind only. Suppose $p$ is a continuous function defined on $V$, then $p$ is sublinear whenever

1) $p(x+y) \leq p(x)+p(y)$ for all $x, y \in V$;

2) $p(c x)=c p(x)$ for all $x \in V, c \in \mathbf{R}_{+}$.

So if $p_{1}, p_{2}$ are sublinear functions, then $p_{1}+p_{2}$ is a sublinear function.

We shall say that a function $f \in \mathcal{F}\left(\mathbf{C}^{n}\right)$ is simple, if its dequantization $\hat{f}$ exists and a.e. coincides with a sublinear function; by misuse of language, we shall denote this (uniquely defined everywhere on $V$ ) sublinear function by the same symbol $\hat{f}$. 
Recall that simple functions $f$ and $g$ are in general position if $\hat{f}(x) \neq \hat{g}(x)$ for all $x$ belonging to an open dense subset of $V$. In particular, generalized monomials are in general position whenever they are linearly independent.

Denote by $\operatorname{Sim}\left(\mathbf{C}^{n}\right)$ the set of all simple functions defined on $V$ and denote by $\operatorname{Sim}_{+}\left(\mathbf{C}^{n}\right)$ the set $\operatorname{Sim}\left(\mathbf{C}^{n}\right) \cap \mathcal{D}_{+}\left(\mathbf{C}^{n}\right)$. By $\operatorname{Sbl}(V)$ denote the set of all (continuous) sublinear functions defined on $V=\mathbf{R}^{n}$ and by $\operatorname{Sbl}_{+}(V)$ denote the image $\widehat{\operatorname{Sim}_{+}}\left(\mathbf{C}^{n}\right)$ of $\operatorname{Sim}_{+}\left(\mathbf{C}^{n}\right)$ under the dequantization transform.

The following statements can be easily deduced from Theorem 1 and definitions.

Corollary 3. The set $\operatorname{Sim}_{+}\left(\mathbf{C}^{n}\right)$ is a subsemiring of $\mathcal{D}_{+}\left(\mathbf{C}^{n}\right)$ and $\operatorname{Sbl}_{+}(V)$ is an idempotent subsemiring of $\widehat{\mathcal{D}_{+}}(V)$. The dequantization transform generates an epimorphism of $\operatorname{Sim}_{+}\left(\mathbf{C}^{n}\right)$ onto $S_{b l}(V)$. The set $S b l(V)$ is an idempotent semiring with respect to the operations $(f \oplus g)(x)=\max \{f(x), g(x)\},(f \odot g)(x)=f(x)+g(x)$.

Corollary 4. Polynomials and generalized polynomials are simple functions.

We shall say that functions $f, g \in \mathcal{D}(V)$ are asymptotically equivalent whenever $\hat{f}=\widehat{g}$; any simple function $f$ is an asymptotic monomial whenever $\hat{f}$ is a linear function. A simple function $f$ will be called an asymptotic polynomial whenever $\hat{f}$ is a sum of a finite collection of nonequivalent asymptotic monomials.

Corollary 5. Every asymptotic polynomial is a simple function.

ExAmple 1. Generalized polynomials, logarithmic functions of (generalized) polynomials, and products of polynomials and logarithmic functions are asymptotic polynomials. This follows from our definitions and formula (2).

\section{SubdifFERENTIALS OF SUBLinEAR FUnCTIONS}

We shall use some elementary results from convex analysis. These results can be found, e.g., in [5, ch. $1, \S 1$.

For any function $p \in S b l(V)$ we set

$$
\partial p=\{v \in V \mid(v, x) \leq p(x) \forall x \in V\} .
$$

It is well known from convex analysis that for any sublinear function $p$ the set $\partial p$ is exactly the subdifferential of $p$ at the origin. The following propositions are also known in convex analysis.

Proposition 2. Suppose $p_{1}, p_{2} \in S b l(V)$, then

1) $\partial\left(p_{1}+p_{2}\right)=\partial p_{1} \odot \partial p_{2}=\left\{v \in V \mid v=v_{1}+v_{2}\right.$, where $\left.v_{1} \in \partial p_{1}, v_{2} \in \partial p_{2}\right\}$;

2) $\partial\left(\max \left\{p_{1}(x), p_{2}(x)\right\}\right)=\partial p_{1} \oplus \partial p_{2}$.

Recall that $\partial p_{1} \oplus \partial p_{2}$ is a convex hull of the set $\partial p_{1} \cup \partial p_{2}$.

Proposition 3. Suppose $p \in S b l(V)$. Then $\partial p$ is a nonempty convex compact subset of $V$. 
Corollary 6. The map $p \mapsto \partial p$ is a homomorphism of the idempotent semiring $S b l(V)$ (see Corollary 3) to the idempotent semiring $\mathcal{S}$ of all convex compact subsets of $V$ (see Section 1 above).

\section{NeWTON SETS FOR SIMPLE FUnCTIONS}

For any simple function $f \in \operatorname{Sim}\left(\mathbf{C}^{n}\right)$ let us denote by $N(f)$ the set $\partial(\hat{f})$. We shall call $N(f)$ the Newton set of the function $f$.

Proposition 4. For any simple function $f$, its Newton set $N(f)$ is a nonempty convex compact subset of $V$.

This proposition follows from Proposition 3 and definitions.

Theorem 2. Suppose that $f$ and $g$ are simple functions. Then

1) $N(f g)=N(f) \odot N(g)=\left\{v \in V \mid v=v_{1}+v_{2}\right.$ with $\left.v_{1} \in N(f), v_{2} \in N(g)\right\}$;

2) $N(f+g)=N(f) \oplus N(g)$, if $f_{1}$ and $f_{2}$ are in general position or $f_{1}, f_{2} \in \operatorname{Sim}_{+}\left(\mathbf{C}^{n}\right)$ (recall that $N(f) \oplus N(g)$ is the convex hull of $N(f) \cup N(g)$ ).

This theorem follows from Theorem 1, Proposition 2 and definitions.

Corollary 7. The map $f \mapsto N(f)$ generates a homomorphism from $\operatorname{Sim}_{+}\left(\mathbf{C}^{n}\right)$ to $\mathcal{S}$.

This statement follows from Theorem 1, Corollary 1, Corollary 6, and Theorem 2.

Proposition 5. Let $f=m_{a, d}(x)=a \prod_{i=1}^{n} x_{i}^{d_{i}}$ be a monomial; here $d=\left(d_{1}, \ldots, d_{n}\right) \in$ $V=\mathbf{R}^{n}$ and $a$ is a nonzero complex number. Then $N(f)=\{d\}$.

This follows from Proposition 1, Corollary 2 and definitions.

Corollary 8. Let $f=\sum_{d \in D} m_{a_{d}, d}$ be a polynomial. Then $N(f)$ is the polytope $\oplus_{d \in D}\{d\}$, i.e. the convex hull of the finite set $D$.

This statement follows from Theorem 2 and Proposition 5. Thus in this case $N(f)$ is the well-known classical Newton polytope of the polynomial $f$.

Now the following corollary is obvious.

Corollary 9. Let $f$ be a generalized or asymptotic polynomial. Then its Newton set $N(f)$ is a convex polytope.

Example 2. Consider the one dimensional case, i.e., $V=\mathbf{R}$ and suppose $f_{1}=$ $a_{n} x^{n}+a_{n-1} x^{n-1}+\cdots+a_{0}$ and $f_{2}=b_{m} x^{m}+b_{m-1} x^{m-1}+\cdots+b_{0}$, where $a_{n} \neq 0, b_{m} \neq 0$, $a_{0} \neq 0, b_{0} \neq 0$. Then $N\left(f_{1}\right)$ is the segment $[0, n]$ and $N\left(f_{2}\right)$ is the segment $[0, m]$. So the map $f \mapsto N(f)$ corresponds to the map $f \mapsto \operatorname{deg}(f)$, where $\operatorname{deg}(f)$ is a degree of the polynomial $f$. In this case Theorem 2 means that $\operatorname{deg}(f g)=\operatorname{deg} f+\operatorname{deg} g$ and $\operatorname{deg}(f+g)=\max \{\operatorname{deg} f, \operatorname{deg} g\}=\max \{n, m\}$ if $a_{i} \geq 0, b_{i} \geq 0$ or $f$ and $g$ are in general position.

REMARK. The above results can be extended to the infinite-dimensional case. This generalization will be the subject of another paper. 


\section{REFERENCES}

[1] V. P. Maslov, On a new superposition principle for optimization problems, Uspekhi Mat. Nauk, [Russian Math. Surveys] 42 (1987), no. 3, 39-48.

[2] G. L. Litvinov and V. P. Maslov, Correspondence principle for idempotent calculus and some computer applications, (IHES/M/95/33), Institut des Hautes Etudes Scientifiques, Bures-sur-Yvette, 1995. Also: 3], p. 420-443 and arXiv:math.GM/0101021

[3] J. Gunawardena, (Ed.), Idempotency, Publ. of the Newton Institute, Vol. 11, Cambridge University Press, Cambridge, 1998.

[4] G. L. Litvinov, V. P. Maslov, G. B. Shpiz, Idempotent functional analysis: an algebraic approach, Mathematical Notes 69 (2001), no. 5, 696-729. Also arXiv:math.FA/0009128.

[5] G. G. Magaril-Il'yaev and V. M. Tikhomirov, Convex analysis: theory and applications, Translations of Mathematical Monographs, vol. 222, Amer. Math. Soc., Providence, RI, 2003.

[6] R. T. Rockafellar, Convex analysis, Princeton Univ. Press, Princeton, 1970.

Independent University of Moscow, 11 Bol'shoi Vlasievskit Per., Moscow 121002, Russia

E-mail address: islc@dol.ru

Moscow Center for Continuous Mathematical Education, 11 Bol'shoi Vlasievski Per., Moscow 121002, Russia 